\title{
Two-Mode Eddy-Viscosity Compressible Turbulence Model for Supercritical Fluid
}

\author{
Bofeng Bai \\ Xi'an Jiaotong University \\ Xi'an, 710049, China \\ bfbai@mail.xjtu.edu.cn
}

\begin{abstract}
High compressibility in the pseudo-critical region of the supercritical fluid brings big challenges to the modeling of turbulence, and the existing models cannot produce accurate predictions. In this talk, the turbulent flow characteristic of supercritical fluid are summarized and then Helmholtz decomposition is employed for the modification of the compressible turbulence model and for the first time a "two-mode" eddy-viscosity compressible turbulence model (TM-EVM) for supercritical fluid has been proposed. We decompose the supercritical fluid turbulence into the solenoidal mode and the compressive mode, and then the mathematical framework of the compressive mode of turbulence is established, including the transport equations of the first-order moments and the second-order moments. The solution of the energy spectrum is accordingly obtained and the modified eddy viscosity formula in the $\mathrm{k}-\varepsilon$ model considering the compressibility of supercritical fluid turbulence is proposed. We have tested the TM-EVM on the tube flow and the results show that it accurately describes the turbulent heat transfer characteristics in the pseudo-critical region. Moreover, the predictions on the statistics of the shear turbulence collapse well with the DNS data, including the mean parameters, the statistics and the TKE budget. Based on this model, we find that the turbulent kinetic energy obviously increases while the turbulent dissipation rate changes slightly in the pseudo-critical region compared with what is predicted by the existing models, indicating that compressibility plays an important role for the large-scale motion but has negligible effect on the small scale. We also find that the anisotropic part of the Reynolds shear stress approximately remains constant in the logarithmic layer and this phenomenon is independent of the Reynolds number, which means that the equilibrium layer exists even in the supercritical fluid turbulence with constant density fluctuations.
\end{abstract}

